\section{CS-07 ECONOMIC EVALUATION OF DAMAGE ACCRUAL IN AN INTERNATIONAL SLE INCEPTION COHORT}

${ }^{1}$ Ann E Clarke*, ${ }^{2}$ lan N Bruce, ${ }^{3}$ Murray B Urowitz, ${ }^{4} J o h n$ G Hanly, Juanita Romero-Diaz, ${ }^{6}$ Caroline Gordon, ${ }^{7}$ Sang-Cheol Bae, ${ }^{8}$ Sasha Bernatsky, ${ }^{9}$ Daniel J Wallace, ${ }^{10}$ Joan T Merrill, ${ }^{11}$ David A Isenberg, ${ }^{11}$ Anisur Rahman, ${ }^{12}$ Ellen M Ginzler, ${ }^{13}$ Michelle Petri, ${ }^{14}$ MA Dooley, ${ }^{15}$ Paul Fortin, ${ }^{2}$ Dafna D Gladman, ${ }^{2} J o r g e$ Sanchez-Guerrero, ${ }^{16}$ Kristjan Steinsson, ${ }^{17}$ Rosalind Ramsey-Goldman, ${ }^{18}$ Munther A Khamashta, ${ }^{19}$ Cynthia Aranow, ${ }^{20}$ Graciela $S$ Alarcón, ${ }^{21}$ Susan Manzi, ${ }^{22}$ Ola Nived, ${ }^{23}$ Asad A Zoma, ${ }^{24}$ Ronald $F$ van Vollenhoven, ${ }^{25}$ Manuel Ramos-Casals, ${ }^{26}$ Guillermo Ruiz-Irastorza, ${ }^{27} \mathrm{~S}$ Sam Lim, ${ }^{28}$ Kenneth C Kalunian, ${ }^{29}$ Murat Inanc, ${ }^{30}$ Diane L Kamen, ${ }^{31}$ Christine A Peschken, ${ }^{32}$ Soren Jacobsen, ${ }^{33}$ Anca Askanase, ${ }^{34}$ Yvan St Pierre, ${ }^{35}$ Li Su, ${ }^{35}$ Vernon Farewell. ${ }^{1}$ Division of Rheumatology, Cumming School of Medicine, University of Calgary, Alberta, Canada; ${ }^{2}$ Arthritis Research UK Epidemiology Unit, Institute of Inflammation and Repair, Manchester Academic Health Sciences Centre, The University of Manchester, Manchester, UK; and NIHR Manchester Musculoskeletal Biomedical Research Unit, Central Manchester University Hospitals NHS Foundation Trust, Manchester Academic Health Science Centre; ${ }^{3}$ Centre for Prognosis Studies in the Rheumatic Diseases, Toronto Western Hospital and University of Toronto, Ontario, Canada; ${ }^{4}$ Division of Rheumatology, Department of Medicine and Department of Pathology, Queen Elizabeth II Health Sciences Centre and Dalhousie University, Halifax, Nova Scotia, Canada; ${ }^{5}$ Instituto Nacional de Ciencias Medicas y Nutrición, Mexico City, Mexico; ${ }^{6}$ Rheumatology Research Group, School of Immunity and Infection, College of Medical and Dental Sciences, University of Birmingham, Birmingham, UK; ${ }^{7}$ Department of Rheumatology, Hanyang University Hospital for Rheumatic Diseases, Seoul, Korea; ${ }^{8}$ Divisions of Rheumatology and Clinical Epidemiology, McGill University Health Centre; Montreal, Quebec, Canada; ${ }^{9}$ Cedars-Sinai/David Geffen School of Medicine at UCLA, Los Angeles, CA, USA; ${ }^{10}$ Department of Clinical Pharmacology, Oklahoma Medical Research Foundation, OKlahoma City, OK, USA; ${ }^{11}$ Centre for Rheumatology, Department of Medicine, University College London, UK; ${ }^{12}$ Department of Medicine, SUNY Downstate Medical Center, Brooklyn, NY, USA; ${ }^{13}$ Division of Rheumatology, Johns Hopkins University School of Medicine, Baltimore, MD, USA; ${ }^{14}$ Thurston Arthritis Research Center, University of North Carolina, Chapel Hill, NC, USA; ${ }^{15}$ Division of Rheumatology, Centre Hospitalier Universitaire de Québec et Université Laval, Quebec City, Canada; ${ }^{16}$ Center for Rheumatology Research, Landspitali University hospital, Reykjavik, Iceland; ${ }^{17}$ Northwestern University and Feinberg School of Medicine, Chicago, IL, USA; ${ }^{18}$ Lupus Research Unit, The Rayne Institute, St Thomas' Hospital, King's College London School of Medicine, UK, London, UK; ${ }^{19}$ Feinstein Institute for Medical Research, Manhasset, NY, USA; ${ }^{20}$ Department of Medicine, University of Alabama at Birmingham, Birmingham, AL, USA; ${ }^{21}$ Alleghany Health Network, Pittsburgh, PA, USA; ${ }^{22}$ Department of Rheumatology, University Hospital Lund, Lund, Sweden; ${ }^{23}$ Lanarkshire Centre for Rheumatology, Hairmyres Hospital, East Kilbride, UK; ${ }^{24}$ University of Amsterdam, Rheumatology and Immunology Center, Amsterdam, Noord-Holland, NL; ${ }^{25}$ Josep Font Autoimmune Diseases Laboratory, IDIBAPS, Department of Autoimmune Diseases, Hospital Clínic, Barcelona, Spain; ${ }^{26}$ Autoimmune Diseases Research Unit, Department of Internal Medicine, BioCruces Health Research Institute, Hospital Universitario Cruces, University of the Basque Country, Barakaldo, Spain; ${ }^{27}$ Emory University School of Medicine, Division of Rheumatology, Atlanta, Georgia, USA; ${ }^{28}$ UCSD School of Medicine, La Jolla, CA, USA; ${ }^{29}$ Division of Rheumatology, Department of Internal Medicine, Istanbul Medical Faculty, Istanbul University, Istanbul, Turkey; ${ }^{30}$ Medical University of South Carolina, Charleston, South Carolina, USA; ${ }^{31}$ University of Manitoba, Winnipeg, Manitoba, Canada; ${ }^{32}$ Department of Rheumatology, Rigshospitalet, Copenhagen University Hospital, Copenhagen, Denmark; ${ }^{33}$ Hospital for Joint Diseases, NYU, Seligman Centre for Advanced Therapeutics, New York NY; ${ }^{34}$ Research Institute of McGill University Health Centre, Montreal, Quebec, Canada; ${ }^{35}$ MRC Biostatistics Unit, Institute of Public Health, University Forvie Site, Cambridge, UK

10.1136/lupus-2018-Ism.42
Background Little is known about the association of healthcare costs with damage accrual in SLE. We describe the costs associated with damage progression using multi-state modeling.

Methods Patients fulfilling the revised ACR Classification Criteria for SLE from 32 centres in 11 countries were enrolled in the Systemic Lupus International Collaborating Clinics (SLICC) inception cohort within 15 months of diagnosis. Annual data on demographics, SLE disease activity (SLEDAI$2 \mathrm{~K}$ ), damage (SLICC/ACR Damage Index [SDI] if $\geq 6$ months from diagnosis), hospitalizations, medications, dialysis, and utilization of selected medical/surgical procedures were collected. Annual health resource utilization was costed using 2017 Canadian prices. Annual costs associated with SDI states were obtained from multiple regressions adjusting for age, sex, race/ ethnicity, and disease duration. As there were relatively few transitions to SDI states $5-11$, these were merged into a single SDI state. Five and 10 year cumulative costs were estimated by multiplying annual costs associated with each SDI state by the expected duration in each state, which was forecasted using a multi-state model and longitudinal SDI data from the SLICC Inception Cohort (Bruce IN et al. Ann Rheum Dis 2015;74:1706-13). Future costs were discounted at a yearly rate of $3 \%$.

Results 1676 patients participated, $88.7 \%$ female, $49.2 \%$ Caucasian, mean age at diagnosis 34.6 years (SD 13.4), mean disease duration at enrollment 0.5 years (range $0-1.3$ years), and mean follow up 7.8 years (range 0.6-16.9 years). Health resource utilization and annual costs (after adjustment using regression) were markedly higher in those with higher SDIs (SDI $=0$, annual costs $\$ 1847,95 \% \mathrm{CI} \$ 1120$ to $\$ 2574$; SDI $\geq 5$, annual costs $\$ 26772$, 95\% CI $\$ 19631$ to $\$ 33813$ ). At $\mathrm{SDI} \leq 2$, hospitalizations and medications accounted for $97.1 \%$ of direct costs, whereas at $\mathrm{SDI} \geq 3$, dialysis was responsible for $55.0 \%$.

Five and 10 year cumulative costs stratified by baseline SDI were calculated by multiplying the annual costs associated with each SDI by the expected duration in that state. Five and 10 year costs were greater in those with the highest SDIs at baseline (table 1).

Conclusions Patients with the highest baseline SDIs incur annual costs and 10 year cumulative costs that are at least 10fold higher than those with the lowest baseline SDI. By estimating the expected duration in each SDI state and incorporating annual costs, disease severity at presentation can be used to predict future healthcare costs, critical knowledge for cost-effectiveness evaluations of novel therapies.

Acknowledgements The Systemic Lupus International Collaborating Clinics (SLICC) research network received partial funding for this study from UCB Pharmaceuticals.

Abstract CS-07 Table 1 Predicted 5 and 10-year cumulative costs stratified by baseline SDI

\begin{tabular}{|c|c|c|c|c|}
\hline \multirow[t]{2}{*}{ Baseline SDI State } & \multicolumn{4}{|c|}{ Healthcare Costs, Mean, 95\% Cl 2017 Canadian \$ } \\
\hline & 5 Year Cumulative Costs & Relative Cost & 10 Year Cumulative Costs & Relative Cost \\
\hline 0 & $9692(5834,13$ 551) & 1 & $21986(13454,30$ 518) & 1 \\
\hline 1 & $21788(15380,28$ 197) & 2.2 & $54813(38989,70$ 637) & 2.5 \\
\hline 2 & $34825(25564,44087)$ & 3.6 & 88361 (64 498, 112 223) & 4.0 \\
\hline 3 & $59254(43746,74$ 762) & 6.1 & $136068(99690,172$ 446) & 6.2 \\
\hline 4 & $93617(68$ 104, 119 129) & 9.7 & $193549(140889,246$ 208) & 8.8 \\
\hline$\geq 5$ & $124998(91636,158360)$ & 12.9 & 230859 (168 871, 292846$)$ & 10.5 \\
\hline
\end{tabular}

